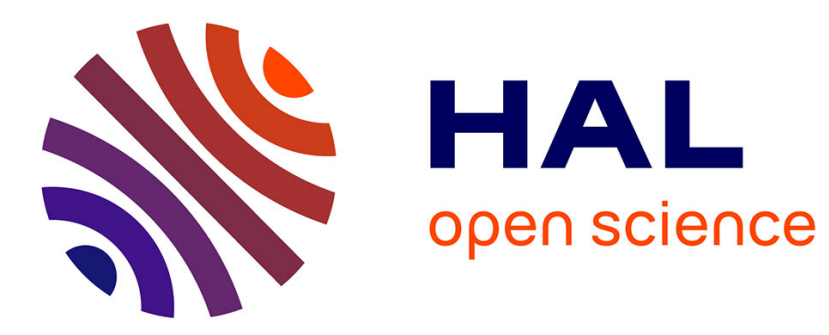

\title{
Efficient Parallel Transport of Deformations in Time Series of Images: from Schild's to Pole Ladder
}

\author{
Marco Lorenzi, Xavier Pennec
}

\section{To cite this version:}

Marco Lorenzi, Xavier Pennec. Efficient Parallel Transport of Deformations in Time Series of Images: from Schild's to Pole Ladder. Journal of Mathematical Imaging and Vision, 2013, 50 (1-2), pp.5-17. 10.1007/s10851-013-0470-3 . hal-00870489

\section{HAL Id: hal-00870489 \\ https://hal.inria.fr/hal-00870489}

Submitted on 7 Oct 2013

HAL is a multi-disciplinary open access archive for the deposit and dissemination of scientific research documents, whether they are published or not. The documents may come from teaching and research institutions in France or abroad, or from public or private research centers.
L'archive ouverte pluridisciplinaire HAL, est destinée au dépôt et à la diffusion de documents scientifiques de niveau recherche, publiés ou non, émanant des établissements d'enseignement et de recherche français ou étrangers, des laboratoires publics ou privés. 


\title{
Efficient Parallel Transport of Deformations in Time Series of Images: from Schild's to Pole Ladder
}

\author{
Marco Lorenzi • Xavier Pennec • for the \\ Alzheimer's Disease Neuroimaging Initiative
}

the date of receipt and acceptance should be inserted later

\begin{abstract}
Group-wise analysis of time series of images requires to compare longitudinal evolutions of images observed on different subjects. In medical imaging, longitudinal anatomical changes can be modeled thanks to non-rigid registration of follow-up images. The comparison of longitudinal trajectories requires the transport (or "normalization") of longitudinal deformations in a common reference frame. We previously proposed an effective computational scheme based on the Schild's ladder for the parallel transport of diffeomorphic deformations parameterized by tangent velocity fields, based on the construction of a geodesic parallelogram on a manifold. Schild's ladder may be however inefficient for transporting longitudinal deformations from image time series of multiple time points, in which the computation of the geodesic diagonals is required several times. We propose here a new algorithm, the pole ladder, in which one diagonal of the parallelogram is the baseline-to-reference frame geodesic. This drastically reduces the number of geodesics to compute. Moreover, differently from the Schild's ladder, the pole ladder is symmetric with respect to the baseline-to-reference frame geodesic. From the theoretical point of view, we show that the pole ladder is rigorously equivalent to the Schild's ladder when transporting along geodesics. From the practical point of view, we establish the computational advantages and demonstrate the effectiveness of this very simple method by comparing with standard methods of transport on simulated images with progressing brain atrophy. Finally, we illustrate its application to a clinical problem: the measurement of the longitudinal progression
\end{abstract}

This work was partially funded by the European Research Council (ERC advanced Grant MedYMA), ANR blanc Karametria and the EU project Care4Me.

Data used in preparation of this article were obtained from the Alzheimer's Disease Neuroimaging Initiative (ADNI) database (www.loni.ucla.edu/ADNI). As such, the investigators within the ADNI contributed to the design and implementation of ADNI and/or provided data but did not participate in analysis or writing of this report. A complete listing of ADNI investigators can be found at:www.loni.ucla.edu/ADNI/Collaboration/ADNI_Authorship_list.pdf

M. Lorenzi, and X. Pennec

Asclepios Research Project, INRIA Sophia Antipolis, France

Tel.: +334923876 60

Fax.: +33492387669

E-mail: marco.lorenzi@inria.fr, xavier.pennec@inria.fr 
in Alzheimer's disease. Results suggest that an important gain in sensitivity could be expected in group-wise comparisons.

Keywords Parallel Transport · Affine Connection · Riemannian Geometry · Lie Group Theory · Imaging · Non-rigid registration

\section{Introduction}

Modeling the temporal evolution of the tissues of the body is an important goal of medical image analysis, for instance for understanding the structural changes of organs affected by a pathology, or for studying the physiological growth during the life span. For such purposes we need to analyze and compare the observed anatomical differences between follow-up sequences of anatomical images of different subjects. Non-rigid registration is one of the main instruments for modeling anatomical differences from images. The aim of non-rigid registration is to encode the observed structural changes as deformation fields densely represented in the image space, which represent the warping required to match the observed differences. This way, the anatomical changes can be modeled and quantified by analyzing the associated deformations.

\subsection{Normalizing Longitudinal Deformations in a Common Reference Frame}

We can identify two distinct settings for the application of non-rigid registration: longitudinal and cross-sectional. In the former, non-rigid registration estimates the deformation field which explains the longitudinal anatomical (intrasubject) changes that usually reflect biological phenomena of interest, like atrophy or growth. In the latter, the deformation field accounts for the anatomical differences between different subjects (inter-subject), in order to match homologous anatomical regions. These two settings are profoundly different, since in the cross-sectional setting we want to compare different anatomies which might have different topologies. In this case the deformations are often a scale of magnitude higher than the ones characterizing the usually subtle variations of the longitudinal setting.

In case of group-wise analysis of longitudinal deformations, longitudinal and cross-sectional settings must be integrated in a consistent manner. In fact, the comparison of longitudinal deformations is usually performed after normalizing them in a common reference frame through the inter-subject registration, and the choice of the normalization method might have a deep impact on the following analysis. In order to preserve and accurately quantify longitudinal deformations in a reference frame space, a rigorous and reliable procedure need thus to be defined.

Normalization of longitudinal deformations can be done in different ways, depending on the analyzed feature. For instance, the scalar Jacobian determinant of longitudinal deformations represents the associated local volume change, and can be compared by scalar resampling in a common reference frame via inter-subject registration. This simple transport of scalar quantities is the basis of the classical deformation/tensor based morphometry techniques [3, 20].

If we consider vector-values characteristics of deformations instead of scalar quantities, the transport is not uniquely defined anymore. For instance, a simple 
method of transport consists in reorienting the longitudinal intra-subject displacement vector field by the Jacobian matrix of the subject-to-reference deformation. Another intuitive method was proposed by [19] and uses the transformation conjugation (change of coordinate system) in order to compose the longitudinal intrasubject deformation with the subject-to-reference one. As pointed out in [7], this practice could potentially introduce variations in the transported deformation and relies on the inverse consistency of the estimated deformations, which can raise numerical problems for large deformations.

\subsection{Parallel Transport in Diffeomorphic Registration}

Among these different normalization methods, the parallel transport of longitudinal deformations is a powerful and promising tool which can be used within the "diffeomorphic registration" setting, thanks to its rich mathematical background. Mathematically, parallel transporting a vector $X$ along a curve $\gamma(t)$ consists in translating it across the tangent spaces to the curve by preserving its parallelism, according to a given derivative operation called (affine) connection, and indicated with $\nabla_{\gamma(t)}(X)$. The connection $\nabla$ is a bilinear function which maps tangent vectors across nearby tangent spaces, and a vector is parallel transported along a curve if $\nabla_{\gamma(t)}(X)=0$, for all $t$.

A first formulation of diffeomorphic registration was proposed with the "Large Deformation Diffeomorphic Metric Mapping (LDDMM)" setting [22,5]. In this framework the images are registered by minimizing the length of the trajectory of transformations in the space of diffeomorphism, once specified an opportune right invariant metric. The solution is the endpoint of the flow of a time-varying velocity field, which is a geodesic parameterized through the Riemannian exponential. The LDDMM deformations are thus Riemannian (metric) geodesics, which are also geodesics of the Levi-Civita connection, which is the unique torsion-free connection which preserves the metric ${ }^{1}$

Since LDDMM is generally computationally intensive, a different diffeomorphic registration method was later proposed with the stationary velocity field (SVF) setting [2. In this case the diffeomorphisms are parameterized by stationary velocity fields through the Lie group exponential. The restriction to stationary velocity fields simplifies the registration problem and provides efficient numerical schemes for the computation of deformations. This time the flow associated to SVFs is a one-parameter subgroup, which is a geodesic with respect to the Cartan-Shouten connections [16, 14]. One-parameter subgroups are generally not metric geodesics, since there do not exist any left and right invariant metric on non-compact and non-commutative groups.

In both the LDDMM and SVF settings, the longitudinal deformation is encoded by the initial tangent velocity field. The transport of longitudinal deformations can be then naturally formulated as the parallel transport of tangent vectors along geodesics according to the underlying connection, i.e. the Levi-Civita connection in LDDMM, and the canonical symmetric Cartan-Shouten connection in the SVF setting.

\footnotetext{
1 For a more detailed discussion we refer to classical books on Riemannian geometry, for example to [8].
} 
As illustrated in abstract form in [1, parallel translation can be approximated infinitesimally by Jacobi fields. Following this intuition, a computational method for the parallel transport along geodesics of the right invariant metric was proposed in the LDDMM context by 24. This framework enables to transport diffeomorphic deformations of point supported and image data, and it was applied to study the hippocampal shape changes in Alzheimer's disease [18,17]. Although it represents a rigorous implementation of the parallel transport, it comes to the price of the computationally intense scheme for computing geodesics. More importantly, it is limited to the transport along geodesics of the right invariant metric, and does not allow to specify different metrics for longitudinal and inter-subject registrations. While from the theoretical point of view parallel transporting along a generic curve can be approximated by the parallel transport on piecewise geodesics, the effectiveness of the above methods was shown only on LDDMM geodesics, and no general computational schemes were provided.

The parallel transport in the SVF setting was investigated in [14, in which an explicit formula for the parallel transport with respect to the standard CartanShouten connections (left, right and symmetric) in the case of finite dimensional Lie groups was derived. Then it was proposed to seamlessly apply these formulas in the infinite dimensional case of the diffeomorphic registration of images. Although further investigations would be needed to better understand the impact of generalizing to infinite dimensions the concepts and tools defined for the Lie Group theory in finite dimension, practical examples of parallel transport of longitudinal diffeomorphisms in synthetic and real images with respect to the Cartan-Shouten connections showed to be an effective and simple way to transport tangent SVFs.

\section{Contributions and Paper Structure}

In this paper we focus on explicit discrete algorithms for parallel transport.

More specifically, we contribute the pole ladder, a new general and computationally efficient method for parallel transport derived from the Schild's ladder. The proposed method rely on a solid mathematical background and can be easily used in the LDDMM and SVF registration settings where diffeomorphic transformations are parameterized by their initial tangent vectors.

Although the Schild's ladder was known in gravitation theory for 40 years, there does not seem to be any numerical implementation of this algorithm before 13, in which we turned it into an effective algorithm for the parallel transport of deformation vectors. This manuscript is an extension of a recent conference submission [26], and elaborates over these preliminary works to provide a novel construction called the pole ladder. We show that is is equivalent to the Schild's ladder when transporting along arcs of geodesics, but with the computational advantage of minimizing the number of estimation of geodesics when applied to the parallel transport of time series of deformations over multiple time points.

In Section (3) we detail the mathematical basis of the proposed method, while in Section (4) we show how these theoretical concepts can be effectively translated in the image registration context. Finally in Section (5) the methods are tested on the transport of synthetic longitudinal progressions, and on the statistical analysis of the longitudinal atrophy in the brain of patients affected by Alzheimer's disease. 
3 Schild's and pole ladders for the Parallel Transport of Longitudinal Deformations

Before presenting the pole ladder, we first recall the Schild's ladder and its theoretical basis.

\subsection{The Schild's Ladder}

Schild's ladder is a general method for the parallel transport, introduced in the theory of gravitation in [15] after Schild's similar constructions [21]. The method infinitesimally transports a vector along a given curve through the construction of geodesic parallelograms (Figure 1). The Schild's ladder provides a straightforward method to compute a first order approximation of the parallel transport of a vector along a curve using geodesics only. Let $M$ a manifold and $C$ a curve parametrized by the parameter $\tau$ with $\left.\frac{\partial C}{\partial \tau}\right|_{T_{0}}=\mathbf{u}$, and $\mathbf{A} \in T_{P_{0}} M$, a tangent vector on the curve at the point $P_{0}=C(0)$. Let $P_{1}$ be a point on the curve relatively close to $P_{0}$, i.e. separated by a sufficiently small parameter value $\tau$.

The Schild's ladder computes the parallel transport of $\mathbf{A}$ along the curve $C$ as follows:

1. Define a curve on the manifold parametrized by a parameter $\sigma$ passing through the point $P_{0}$ with tangent vector $\left.\frac{\partial}{\partial \sigma}\right|_{P_{0}}=\mathbf{A}$. Chose a point $P_{2}$ on the curve separated by $P_{0}$ by the value of the parameters $\sigma$. The values of the parameters $\sigma$ and $\tau$ should be chosen in order to construct the Schild's ladder within a single coordinate neighborhood.

2. Let $l$ be the geodesic connecting $P_{2}=l(0)$ and $P_{1}=l(\lambda)$, we choose the "middle point" $P_{3}=l(\lambda / 2)$. Now, let us define the geodesic $r$ connecting the starting point $P_{0}$ and $P_{3}$ parametrized by $\rho$ such that $P_{3}=r(\rho)$. Extending the geodesic at the parameter $2 \rho$ we reach the point $P_{4}$. We can now pick a curve connecting $P_{1}$ and $P_{4}$. The vector $A^{\prime}$ tangent to the curve at the point $P 1$ is the parallel translation of $A$ along $C$.

3. If the distance between the points $P_{0}$ and $P_{1}$ is large, the above construction can be iterated for a sufficient number of small steps.

The algorithmic interest of the Schild's ladder is that it only relies on the geometrical information encoded by the geodesics. Although the geodesics on the manifold are not sufficient to recover all the information about the space properties, such as the torsion of the connection, it has been shown that the Schild's ladder describes the parallel transport with respect to the symmetric part of the connection of the space [10. An intuitive view of that point is that the construction of the above diagram is commutative and can be symmetrized with respect to the points $P_{1}$ and $P_{2}$. If the original connection is symmetric, then this procedure provides a correct linear approximation of the parallel transport of vectors.

\subsection{The Pole Ladder}

We propose here a different construction for the parallel transport of vectors based on geodesics parallelograms. If the curve $C$ is geodesic, then it can be itself one 


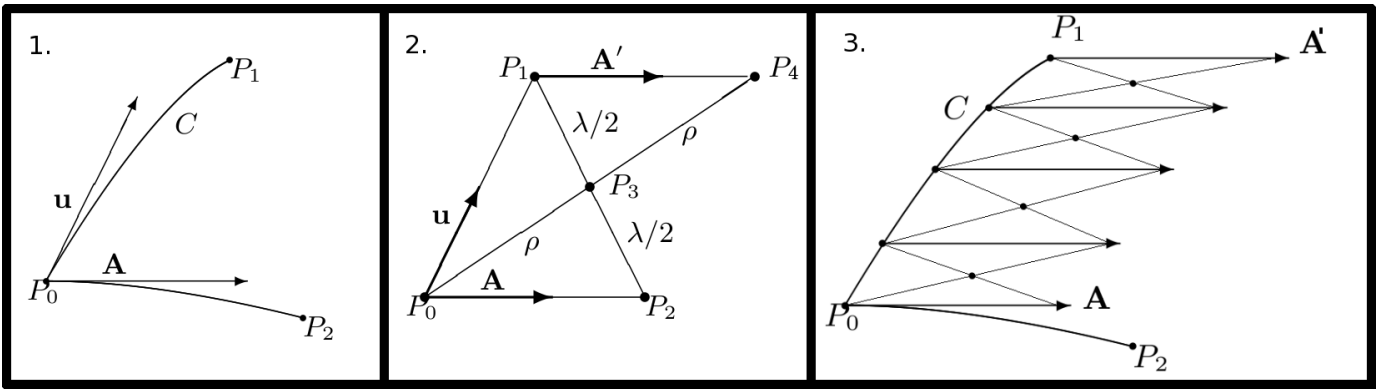

Fig. 1: The Schild' ladder parallel transports a vector $\mathbf{A}$ along the curve $C$ by iterative construction of geodesic parallelograms.

of the diagonals and the Schild's ladder can therefore be adapted by requiring the computation of only one new diagonal of the parallelogram. We define in this way a different ladder scheme, that we name the "pole ladder".

We now prove that the pole ladder is actually realizing the parallel transport. In the diagram of Figure 2 the parallel transport of the tangent vector $v=\dot{C}$ to the geodesic $C$ is specified through the Christoffel symbols $\Gamma_{i j}^{k}$ by the geodesic equation $\dot{\mathbf{v}}+\Gamma_{i j}^{k} \mathbf{v}^{i} \mathbf{v}^{j}=0$. In a sufficiently small neighborhood the relationships can be linearized to give

$$
\mathbf{v}^{k}(t)=\mathbf{v}^{k}(0)-t \Gamma_{i j}^{k}(x(0)) \mathbf{v}^{i}(0) \mathbf{v}^{j}(0)+O\left(t^{2}\right),
$$

and by integrating:

$$
x^{k}(t)=x^{k}(0)+t \mathbf{v}^{k}(0)-\frac{t^{2}}{2} \Gamma_{i j}^{k}(x(0)) \mathbf{v}^{i}(0) \mathbf{v}^{j}(0)+O\left(t^{3}\right) .
$$

By renormalizing the length of the vector $v$ so that $C(-1)=P_{0}, C(0)=M$ and $C(1)=Q_{0}\left(\right.$ and denoting $\left.\Gamma_{i j}^{k}=\Gamma_{i j}^{k}(M)\right)$, we obtain the relations:

$$
\begin{gathered}
P_{0}{ }^{k}=M^{k}-\mathbf{v}_{M}^{k}-\frac{1}{2} \Gamma_{i j}^{k} \mathbf{v}_{M}^{i} \mathbf{v}_{M}^{j}+O\left(\|\mathbf{v}\|^{3}\right), \\
Q_{0}{ }^{k}=M^{k}+\mathbf{v}_{M}^{k}-\frac{1}{2} \Gamma_{i j}^{k} \mathbf{v}_{M}^{i} \mathbf{v}_{M}^{j}+O\left(\|\mathbf{v}\|^{3}\right) .
\end{gathered}
$$

Similarly, we have along the second geodesic:

$$
\begin{gathered}
P_{1}{ }^{k}=M^{k}-\mathbf{u}_{M}^{k}-\frac{1}{2} \Gamma_{i j}^{k} \mathbf{u}_{M}^{i} \mathbf{u}_{M}^{j}+O\left(\|\mathbf{u}\|^{3}\right), \\
Q_{1}{ }^{k}=M^{k}+\mathbf{u}_{M}^{k}-\frac{1}{2} \Gamma_{i j}^{k} \mathbf{u}_{M}^{i} \mathbf{u}_{M}^{j}+O\left(\|\mathbf{u}\|^{3}\right) .
\end{gathered}
$$

Now, to compute the geodesics joining $P_{0}$ to $P_{1}$ and $Q_{0}$ to $Q_{1}$, we have to use a Taylor expansion of the Christoffel symbols $\Gamma_{i j}^{k}$ around the point $M$. In the following, we indicate the coordinate according to which the quantity is derived by the index after a comma: $\Gamma_{i j, a}^{k}=\partial_{a} \Gamma_{i j}^{k}$ :

$$
\Gamma_{i j}^{k}\left(P_{0}\right)=\Gamma_{i j}^{k}+\Gamma_{i j, a}^{k}\left(-\mathbf{v}_{M}^{k}-\frac{1}{2} \Gamma_{i j}^{k} \mathbf{v}_{M}^{i} \mathbf{v}_{M}^{j}\right)+\frac{1}{2} \Gamma_{i j, a b}^{k} \mathbf{v}_{M}^{a} \mathbf{v}_{M}^{b}+O\left(\|\mathbf{v}\|^{3}\right) .
$$


However, the Christoffel symbols are multiplied by a term of order $O\left(\|A\|^{2}\right)$, so that only the first term will be quadratic and all others will be of order 3 with respect to $A$ and $\mathbf{v}_{M}$. Thus, the geodesics joining $P_{0}$ to $P_{1}$ and $Q_{0}$ to $Q_{1}$ have equations:

$$
\begin{aligned}
& P_{1}^{k}=P_{0}^{k}+A^{k}-\frac{1}{2} \Gamma_{i j}^{k} A^{i} A^{j}+O\left(\left(\|A\|+\left\|\mathbf{v}_{M}\right\|\right)^{3}\right), \\
& Q_{1}^{k}=Q_{0}^{k}+B^{k}-\frac{1}{2} \Gamma_{i j}^{k} B^{i} B^{j}++O\left(\left(\|B\|+\left\|\mathbf{v}_{M}\right\|\right)^{3}\right) .
\end{aligned}
$$

Equating $P_{1}^{k}$ in the previous equations gives

$$
\mathbf{u}_{M}^{k}+\frac{1}{2} \Gamma_{i j}^{k} \mathbf{u}_{M}^{i} \mathbf{u}_{M}^{j}=\mathbf{v}_{M}^{k}-A^{k}+\frac{1}{2} \Gamma_{i j}^{k}\left(\mathbf{v}_{M}^{i} \mathbf{v}_{M}^{j}+A^{i} A^{j}\right)+O\left(\left(\|B\|+\left\|\mathbf{v}_{M}\right\|\right)^{3}\right) .
$$

Solving for $\mathbf{u}$ as a second order polynomial in $v_{M}$ and $A$ gives

$$
\mathbf{u}^{k}=\mathbf{v}_{M}^{k}-A^{k}+\frac{1}{2}\left(\Gamma_{i j}^{k}+\Gamma_{j i}^{k}\right) A^{i} \mathbf{v}_{M}^{j}+O\left(\left(\|A\|+\left\|\mathbf{v}_{M}\right\|\right)^{3}\right)
$$

Now equating $Q_{1}^{k}$ in the previous equations gives

$$
B^{k}-\frac{1}{2} \Gamma_{i j}^{k} B^{i} B^{j}=-A^{k}+\left(\Gamma_{i j}^{k}+\Gamma_{j i}^{k}\right) A^{i} \mathbf{v}_{M}^{j}+\frac{1}{2} \Gamma_{i j}^{k} A^{i} A^{j}+O\left(\left(\|A\|+\left\|\mathbf{v}_{M}\right\|\right)^{3}\right) .
$$

Solving for $B^{k}$ as a second order polynomial in $v_{M}$ and $A$ gives:

$$
B^{k}=-A^{k}+\left(\Gamma_{i j}^{k}+\Gamma_{j i}^{k}\right) A^{i} \mathbf{v}^{j}+O\left(\left(\|A\|+\left\|\mathbf{v}_{M}\right\|\right)^{3}\right) .
$$

To verify that this is the correct formula for the parallel transport of $A$, let us observe that the field $A(x)$ is parallel in the direction of $\mathbf{v}^{j}$ if $\nabla_{V} A=0$, i.e. if $\partial_{v} A^{k}+\Gamma_{i j}^{k} A^{i} \mathbf{v}^{j}=0$, which means that $A^{k}(x+\epsilon v)=A^{k}-\epsilon \Gamma_{i j}^{k} A^{i} \mathbf{v}^{j}+O\left(\epsilon^{2}\right)$. If the connection is symmetric, i.e. if $\Gamma_{i j}^{k}=\Gamma_{j i}^{k}$, equation $(1)$ shows that the pole ladder leads to $B^{k} \simeq-A^{k}+2 \Gamma_{i j}^{k} A^{i} \mathbf{v}^{j}$. Thus the pole ladder is realizing the parallel transport for a length $\epsilon=2$ (remember that our initial geodesic was defined from -1 to 1$)$.

We have thus demonstrated that the vector $-B$ of Figure 2 is the transport of $A$ and, due to the locally linear construction, it corresponds necessarily to the one transported by the Schild's ladder.

\section{Application to Images}

Let $I_{i}(i=1 \ldots n)$ be a time series of images with the baseline $I_{0}$ as reference. Consider a template image $T_{0}$, the aim of the procedure is to compute the image $T_{i}$ in order to define the transport of the sequence $I_{0}, \ldots, I_{i}$ in the reference of $T_{0}$. In the sequel, we focus on the transport of a single image $I_{1}$.

To apply the ladders in the context of the images, we define the paths in the space of images by action from the space of diffeomorphism. Let $\mathbb{I}=\left\{f: \mathbb{R}^{3} \rightarrow \mathbb{R}\right\}$ the image space and let us define the action $*: M \times \mathbb{I} \rightarrow \mathbb{I}$ given by $(\varphi, I) \mapsto \varphi * I=$ $I \circ \varphi^{-1}$, where $M$ is the space of the diffeomorphisms. If the distance between two images in the image space is defined in terms of diffeomorphisms [24, then the 


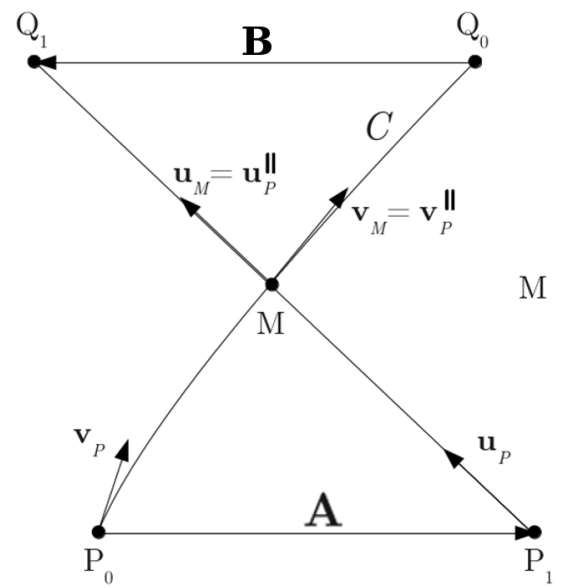

Fig. 2: The pole ladder parallel transports the vector $\mathbf{A}$ along the geodesic $C$. Differently from the Schild's ladder it requires to compute only one diagonal geodesic.

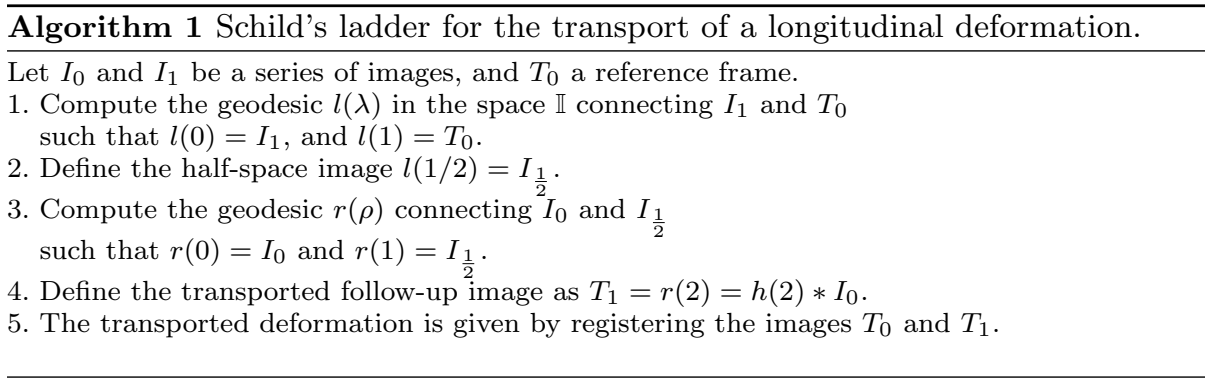

geodesics in the image space are defined by the action of the geodesic paths in the space of the diffeomorphisms.

The Schild's ladder can be naturally translated in the image context (Algorithm 1), by requiring the computation of the two diagonal geodesics $l$ and $r$.

The pole ladder is similar to the Schild's one, with the difference of explicitly using as a diagonal the geodesic $C$ which connects $I_{0}$ and $T_{0}$ (Algorithm 2). This is an interesting property since, given $C$, it requires the computation of only one additional geodesic, thus the transport of time series of several images is based on the same baseline-to-reference curve $C$ (Figure 3 ).

\subsection{Effective Ladders by Using SVFs}

We showed that Schild's and pole ladder are equivalent methods for transporting vectors with geodesic parallelogram. In this paragraph we provide an efficient implementation for the application to images within the SVF setting. Due to the similar nature of the proposed ladders schemes, in the following we refer generally to "ladder" to indicate the two techniques. 


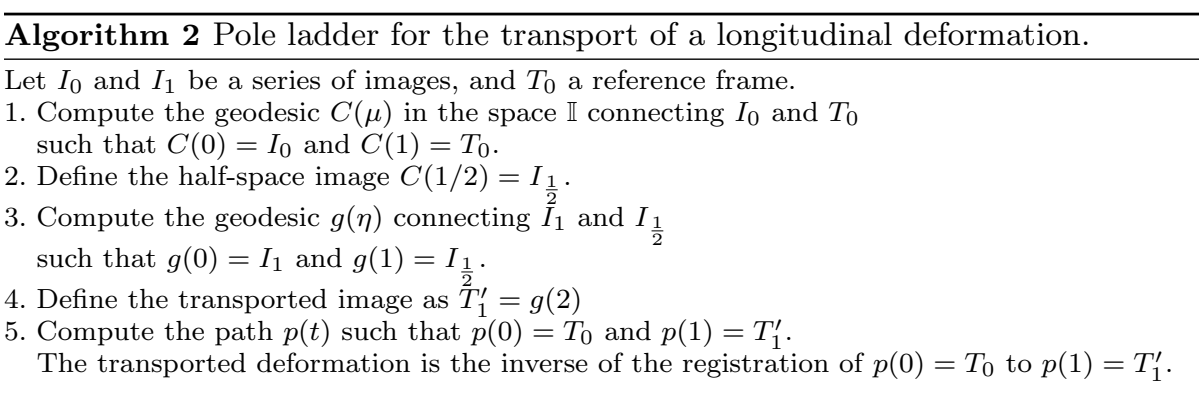

\section{Schild's Ladder}

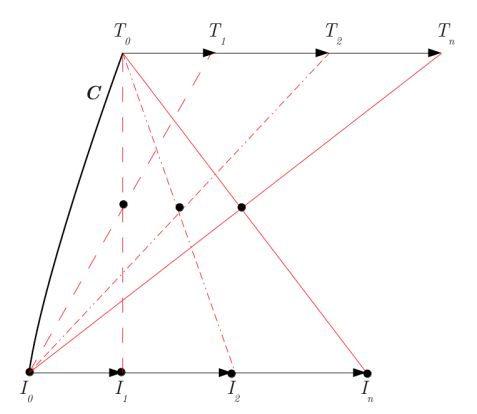

\section{Pole Ladder}

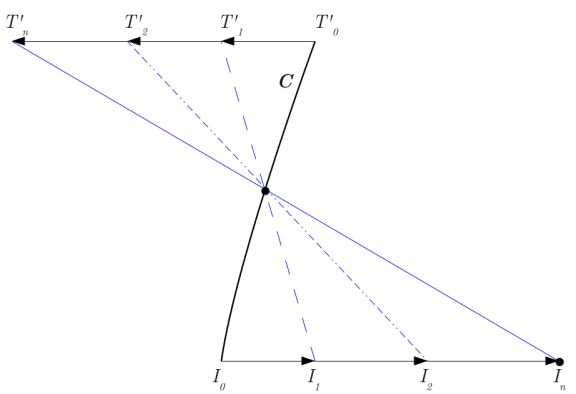

Fig. 3: Geometrical schemes in the Schild's ladder and in the pole ladder. By using the curve $C$ as diagonal, the pole ladder requires the computation of half times of the geodesics (blue) required by the Schild's ladder (red).

Despite the straightforward formulation, algorithms (1) and 22 require multiple evaluations of geodesics in the space of diffeomorphisms and a consequent high cost in terms of computation time and resources.

Moreover, they assume an exact matching, which is bound to lead to important numerical problems. For instance, the definition of $I_{\frac{1}{2}}$ using the forward deformation on $I_{0}$ or the backward from $T_{0}$ leads to very different results. Finally, numerical approximations introduced by exponential and logarithm maps can introduce important errors that can propagate during the iteration of the ladder. For all of these reason, we propose here to reformulate the above scheme in a computationally efficient framework using only transformations.

We use the setting of the SVFs (SVF) diffeomorphic registration as provided for example by the log-Demons [23] algorithm. In particular we base the ladder on the geodesic defined by the Lie group exponential of vector fields. Given a pairs of images $I_{i}, i \in\{0,1\}$, the SVF framework parametrizes the diffeomorphism $\varphi$ required to match the reference $I_{0}$ to the moving image $I_{1}$ by a SVF $u$. The velocity field $u$ is an element of the Lie Algebra $\mathfrak{g}$ of the Lie group of diffeomorphisms $G$, i.e. an element of the tangent space at the identity $T_{i d} G$. The diffeomorphism $\varphi$ belongs to the one parameter subgroup $\varphi=\exp (t u)$ generated by $u$. Notice that we use here the Lie group exponential and not the Riemannian exponential to 
parameterize deformations with SVFs. We can therefore define the paths in the space of the diffeomorphisms from the one parameter subgroup parametrization $l(\lambda)=\exp (\lambda \cdot u)$, and the correspondent paths in the image space.

Computing the ladders in the image space requires a number of interpolations and exponentiations, which could introduce biases due to the numerical approximations. Moreover the registration is constrained to be smooth and it is therefore impossible to reach a perfect match of correspondent intensities in the registered images. We take advantage of the symmetry of the geodesic parallelogram in order to define the following robust scheme.

With reference to Figure (4):

1. Let $I_{1}=\exp (u) * I_{0}$.

2. Compute $v=\operatorname{argmin}_{v \in \mathbb{G}} E\left(T_{0} \circ \exp (-v / 2), I_{0} \circ \exp (v / 2)\right)$, where $E$ is a generic registration energy functional to be minimized.

The half space image $I_{\frac{1}{2}}$ can be defined in terms of $v / 2$ as $\exp (-v / 2) * T_{0}$ or $\exp (v / 2) * I_{0}$. While from the theoretical point of view the two images are identical, the choice of one of them, or even their mean, introduces a bias in the construction. The definition of the half step image can be bypassed by relying on the symmetric construction of the parallelogram.

3. The transformation from $I_{1}$ to $I_{\frac{1}{2}}$ is $\rho=\exp (v / 2) \circ \exp (-u)$ and the symmetry leads to $\exp (\Pi(u))=\exp (v / 2) \circ \rho^{-1}=\exp (v / 2) \circ \exp (u) \circ \exp (-v / 2)$.

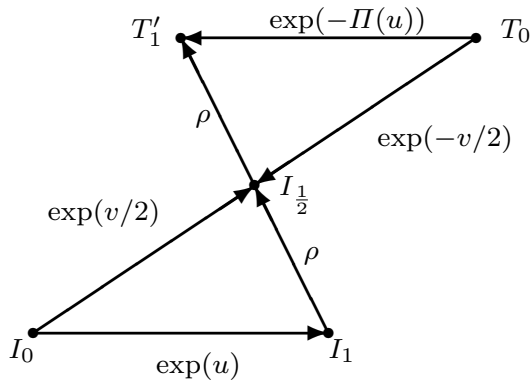

Fig. 4: Ladder with the one parameter subgroups. The transport $\exp (\Pi(u))$ is the deformation $\exp (v / 2) \circ \exp (u) \circ \exp (-v / 2)$

The transport of the deformation $\varphi=\exp (u)$ can be therefore obtained through the conjugate action operated by the deformation parametrized by $v / 2$.

Since the direct computation of the conjugation by composition is potentially biased by the spatial discretization, we propose a numerical scheme to correctly evaluate the transport directly in the Lie Algebra. 
4.2 BCH Formula for the Conjugate Action

The Baker Campbell Hausdorff (BCH) formula was introduced in the SVF diffeomorphic registration in [6] and provides an explicit way to compose diffeomorphisms parameterized by SVFs by operating in the associated Lie Algebra. More specifically, if $v, u$ are SVFs, then $\exp (v) \circ \exp (u)=\exp (w)$ with $w=B C H(v, u)=$ $v+u+\frac{1}{2}[v, u]+\frac{1}{12}[v,[v, u]]-\frac{1}{12}[u,[v, u]]+\ldots$ In particular, for small $u$, the computation can be truncated to any order to obtain a valid approximation for the composition of diffeomorphisms. Applying the truncate $\mathrm{BCH}$ to the conjugate action leads to

$$
\Pi_{B C H}(u) \simeq u+[v / 2, u]+\frac{1}{2}[v / 2,[v / 2, u]]
$$

To establish this formula, let consider the following second order truncation of the $\mathrm{BCH}$ formula

$$
B C H\left((v / 2, u) \simeq v / 2+u+\frac{1}{2}[v / 2, u]+\frac{1}{12}[v / 2,[v / 2, u]]-\frac{1}{12}[u,[v / 2, u]] .\right.
$$

The composition

$$
\Pi_{B C H}(u)=B C H(v / 2, B C H(u,-v / 2))
$$

is

$$
\begin{aligned}
\Pi(u)^{v}= & \underbrace{v / 2+B C H(u,-v / 2)}_{A}+\underbrace{\frac{1}{2}[v / 2, B C H(u,-v / 2)]}_{B} \\
& +\underbrace{\frac{1}{12}[v / 2,[v / 2, B C H(u,-v / 2)]}_{C}-\underbrace{\frac{1}{12}[B C H(u,-v / 2),[v / 2, B C H(u,-v / 2)]]}_{D} .
\end{aligned}
$$

The second order truncation of the four terms is:

$$
\begin{aligned}
A & \simeq u+\frac{1}{2}[u,-v / 2]+\frac{1}{12}[u,[u,-v / 2]]-\frac{1}{12}[-v / 2,[u,-v / 2]], \\
B & \simeq \frac{1}{2}[v / 2, u]+\frac{1}{4}[v / 2,[u,-v / 2]], \\
C & \simeq \frac{1}{12}[v / 2,[v / 2, u]], \\
D & \simeq-\frac{1}{12}[u,[v / 2, u]]+\frac{1}{12}[v / 2,[v / 2, u]] .
\end{aligned}
$$

From the additive and anticommutative properties of the Lie bracket, adding the four terms leads to 2 .

Iterative Computation of the ladder

Once defined the formula for the computation of the ladder, we need a consistent scheme for the iterative construction along trajectories. We recall that the transport by geodesic parallelograms holds only if both sides of the parallelogram are sufficiently small, which in our case means that both longitudinal and inter-subject vectors must be small. This is not the case in practice, since the inter-subject deformation is usually very strong. By definition, the ladder requires to scale down 
vectors to a sufficiently small neighborhood, in order to correctly approximate the transport by parallelograms.

From the theoretical point of view, the degree of approximation of the ladder is proportional to the curvature of the space of deformations. This can be seen by the higher order terms that we dropped off in the proof of Section 3.2 which are all derivatives of the Christoffel symbols. While on a linear space the ladder is the exact parallel transport, when working on curved spaces the error resulting from the non-infinitesimal geodesic parallelogram is proportional to the distance between the points.

From the numerical point of view, we notice that Formula (2) requires the computation of the Lie brackets of the velocity fields. Lie brackets involve the differentiation of the vector which is usually computed on images by finite differences, and which are know to be very sensitive to noise and to be unstable in case of large deformations.

For all these reasons we propose the following iterative scheme based on the properties of SVFs. To provide a sufficiently small vector for the computation of the conjugate we observe that

$$
\begin{aligned}
& \exp (v) \circ \exp (u) \circ \exp (-v)= \\
= & \exp \left(\frac{v}{n}\right) \circ \ldots \circ \exp \left(\frac{v}{n}\right) \circ \exp (u) \circ \exp \left(-\frac{v}{n}\right) \circ \ldots \circ \exp \left(-\frac{v}{n}\right)
\end{aligned}
$$

The conjugation can then be recursively computed in the following way:

1. Scaling step. Find $n$ such that $v / n$ is small.

2. Ladder Step. Compute $w=u+\left[\frac{v}{n}, u\right]+\frac{1}{2}\left[\frac{v}{n},\left[\frac{v}{n}, u\right]\right]$.

3. Let $u=w$.

4. Iterate the steps 2 and $3 n$ times.

The $\mathrm{BCH}$ formula allows to perform the transport directly in the Lie algebra and avoids multiple exponentiation and interpolations, thus reducing the bias introduced by the numerical approximations. Moreover, this method preserves the original "ladder" formulation, operated along the inter-subject geodesic $\exp (t v)$. In fact it iterates the construction of the ladder along the path $\exp (t v)$ over small steps of size $\exp \left(\frac{v}{n}\right)$.

The stability of the proposed method critically depends from the initial scaling step $n$, which determines the step-size of the numerical scheme. Ideally the stepsize should depend on the curvature, and should be therefore small enough in order to minimize the error in case of highly curved space. For this purpose, given the image domain $\Omega$, we define a global scaling factor $n$ in order to guarantee that the given SVF stays sufficiently close to 0 , i.e. in order to satisfy the global condition

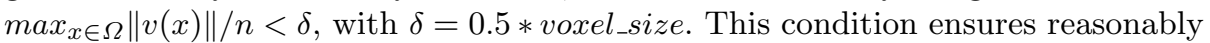
small SVFs, and thus enables the iterative construction of the parallelogram in small neighborhoods.

Section 5.1 shows an example of the impact of the step-size on the transport of longitudinal deformations. 
4.3 Conjugate Action from the Exponential Map

In this section we provide a formula alternative to Formula (2) for computing the transport by conjugate action from the definition of the exponential:

$$
\exp (u)=\lim _{n \rightarrow \infty}\left(\mathrm{Id}+\frac{u}{n}\right)^{n}
$$

We can write:

$$
\exp \left(\Pi_{\text {conj }}(u)\right)=\lim _{n \rightarrow \infty}\left(\exp (v / 2) \circ\left(\operatorname{Id}+\frac{u}{n}\right) \circ \exp (-v / 2)\right)^{n} .
$$

Let $y=\exp (-v / 2)(x)$ and $\phi(x)=\exp (v / 2)(x)$, then

$$
\begin{aligned}
\exp \left(\Pi_{\text {conj }}(u)\right) & =\lim _{n \rightarrow \infty}\left(\phi\left(y+\frac{u(y)}{n}\right)\right)^{n}= \\
& =\lim _{n \rightarrow \infty}\left(\operatorname{Id}+\frac{1}{n}\left(\left.\mathrm{D}(\phi(y))\right|_{\phi^{-1}(x)} \cdot u \circ \phi^{-1}(x)+O\left(\|u\|^{2}\right)\right)\right)^{n} .
\end{aligned}
$$

By the definition of the exponential map, we obtain then a first order approximation for the transported vector given by

$$
\Pi_{\text {conj }}(u)=\left.\mathrm{D}(\exp (v / 2))\right|_{\exp (-v / 2)} \cdot u \circ \exp (-v / 2) .
$$

We note that $\left.\mathrm{D}(\exp (v / 2))\right|_{\exp (-v / 2)}=\mathrm{D}(\exp (-v / 2))^{-1}$. This method provides a formula which enables to compute the transport by reorienting the field $u \circ \exp (-v / 2)$ by the matrix field $\mathrm{D}(\exp (v / 2))$ resampled by $\exp (-v / 2)$, or equivalently, by the matrix field $\mathrm{D}(\exp (-v / 2))^{-1}$.

From a theoretical point of view the results obtained from formula (2) and (3) are equivalent in the continuous domain:

$$
\Pi_{\text {conj }}(u)=u+D(v / 2) \cdot u-D u \cdot v / 2+O\left(\|v\|^{2}\right) \simeq \Pi_{B C H}(u) .
$$

Formula 3 is however potentially more unstable from the numerical point of view, since it requires to interpolate matrix fields which should be computed with specific numerical schemes. For this reason the following experimental section focuses only on the ladders computed with the $\mathrm{BCH}$ scheme of formula (2).

\section{Experiments on Synthetic and Real Data}

\subsection{Impact of the Step-size of the Pole Ladder}

A synthetic longitudinal deformation was created by shrinking a spherical reference image (Figure 5 , left). The longitudinal deformation of the shrinking sphere was transported in an ellipsoidal target space with the pole ladder. The BCH scheme was first directly applied on the inter-subject SVF (step-size = 1), and then iteratively applied as proposed in Section 4.2 by scaling the inter-subject SVF. The condition $\max _{x \in \Omega}\|v(x)\| / n<0.5 \cdot$ voxel_size led to a step-size $n=16$.

The analysis of the log-Jacobian determinant maps associated to the transported deformation shows that when the step-size is large the transport is more 
noisy and unstable (Figure 5 , right). On the contrary, the proposed iterative computation leads to smooth and more stable results. This is confirmed by observing the histogram profile of the log-Jacobian determinant maps. The iterative scheme leads to values that are closer to the ones of the original longitudinal deformation, while when using a large step size the differences are more remarkable.

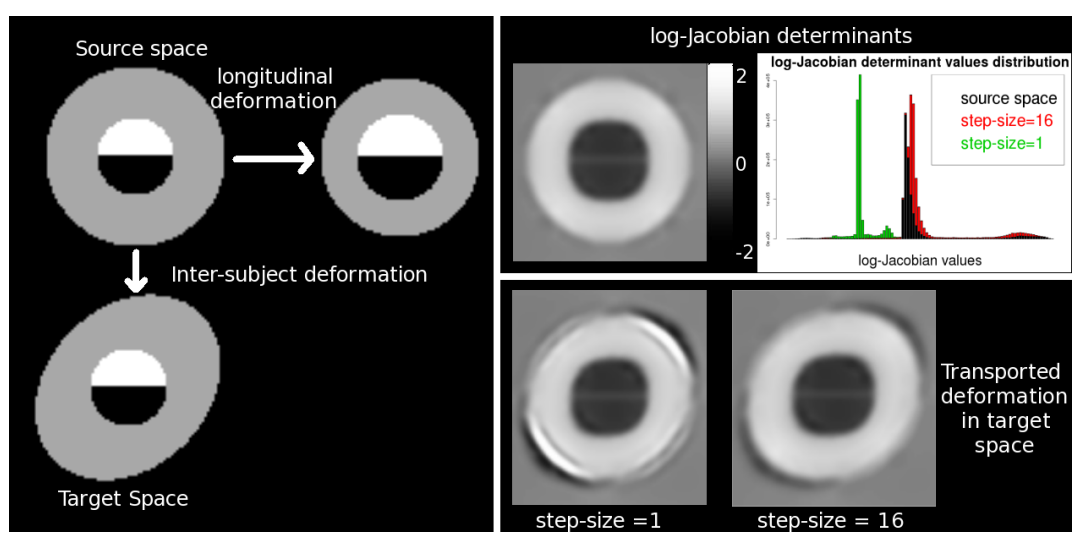

Fig. 5: Impact of the step-size on the pole ladder. Left: the longitudinal deformation of the shrinking ball was transported in the ellipsoidal target space with the pole ladder. The BCH scheme of Section 4.2 was applied 1) directly on the inter-subject SVF (step-size = 1), and 2) iterated by scaling the SVF in order to satisfy the condition $\max _{x \in \Omega}\|v(x)\| / n<\delta$ (resulting step-size =16). Right: The log-Jacobian determinant of the transported deformations shows that a larger step-size leads to more unstable results.

\subsection{Comparison of Different Methods of Transport}

We created a series of simulated deformations based on the deformation field that matches the baseline scan $\left(I_{0}\right)$ of a patient from the ADNI dataset to the 1-year follow-up of the same patient, computed using the LCC-Demons algorithm [11. The ventricular expansion was extracted by masking the corresponding SVF $v$ with a mask including the ventricles. The deformations in the remaining areas of the brain were imposed to be negligible multivariate Gaussian noise. The SVF $v$ was then increasingly scaled $\left(v_{i}=f_{i} v\right.$, with $\left.f_{i}=0.5,1,2,3\right)$ and the resulting deformations fields $\varphi_{i}=\exp \left(v_{i}\right)$ were used to warp the baseline scan $I_{0}$ to generate a longitudinal progression of serial images $I_{i}$ with increasing ventricular expansions.

The longitudinal progression was then transported in five new reference spaces given by the images of five other patients (target space $T_{0}^{k}, k=1, \ldots, 5$ ) along the deformation $\psi_{T}^{k}$ using different methods:

- Schild's and pole ladders (BCH scheme),

- Conjugate Action: $A d_{\psi_{T}^{k}}\left(\varphi_{i}\right)=\psi_{T}^{k(-1)} \circ \varphi_{i} \circ \psi_{T}^{k}$, 


\begin{tabular}{|l|c|c|c|}
\hline & $\begin{array}{c}\text { SVF } \\
(\mathrm{u})\end{array}$ & $\begin{array}{c}\text { Transformation } \\
(\varphi=\exp (u))\end{array}$ & $\begin{array}{c}\text { Scalar Measure } \\
(\mathrm{J}, \log \mathrm{J})\end{array}$ \\
\hline \hline Interpolation of Scalar & No & No & Yes \\
\hline Conjugate Action & No & Yes & Yes \\
\hline Schild's ladder & Yes & Yes & Yes \\
\hline Pole ladder & Yes & Yes & Yes \\
\hline Reorientation & Yes & Yes & Yes \\
\hline
\end{tabular}

Table 1: Different methods of transport and transported features. From the SVF we can infer transformations from which we can extract scalar measures, while the reverse is not possible.

- reorientation of the SVF $v_{i}$ by the Jacobian Matrix of the deformation $\psi_{T}^{k}$ : $J_{\psi_{T}^{k}} v_{i}$

As summarized in Table 1, not all the methods operate on the same features and a direct comparison is not always possible. To test the accuracy of the transport, the different methods were quantitatively compared on the scalar measures representing the amount of change induced in the ventricles. The ventricles masks were segmented for $I_{0}$ and $T_{0}^{k}$ using a semi-automated method [25]. The analyzed features were the average Jacobian and log-Jacobian determinant of the transported deformation, representing respectively the average volume change of the ventricles and the flux of the deformation across the ventricles boundaries (boundary shift) [11].

We note that conjugate action, reorientation, and simple interpolation of scalar fields, are transport methods that are currently majorly employed in applied medical imaging studies $[19,7,9,4$.

\subsection{Results}

In Figure 6. we see an example of synthetic time series of images transported by our pole ladder. The series is consistent with the original trajectory of ventricular expansion while adapting to the new reference.

Figure 7 compares the log-Jacobian scalar image derived from the different methods for a sample subject. The Conjugate method and the Reorientation led to more noisy maps, while for the pole and Schild's ladder (BCH scheme) the resulting Jacobian map adapts to the new reference space while remaining sufficiently smooth, consistently with the simple scalar interpolation of the original log-Jacobian map in the target space.

Table 2 shows the amount of changes measured with the different methods. We note that the pole ladder provides in most of the cases results similar to those obtained by the simple scalar interpolation, and generally very close to the ones measured in the original reference. 
5.4 One Year Follow-up Changes on Alzheimer's Disease

\subsubsection{Experimental Data.}

Data used in the preparation of this article were obtained from the Alzheimer's Disease Neuroimaging Initiative (ADNI) database (adni.loni.ucla.edu). The ADNI was launched in 2003 by the National Institute on Aging (NIA), the National Institute of Biomedical Imaging and Bioengineering (NIBIB), the Food and Drug Administration (FDA), private pharmaceutical companies and non-profit organizations, as a $\$ 60$ million, 5-year public-private partnership. The Principal Investigator of this initiative is Michael W. Weiner, MD, VA Medical Center and University of California - San Francisco. ADNI is the result of efforts of many coinvestigators

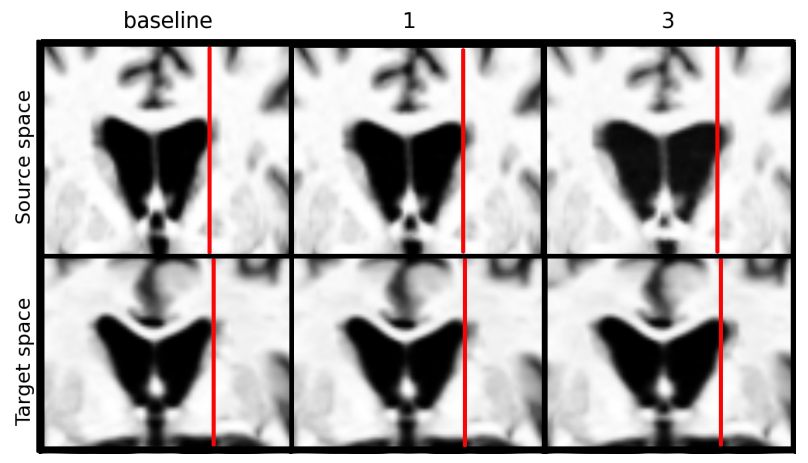

Fig. 6: Transport of time series of images. Top row: original longitudinal trajectory for the ventricular expansion at the different scaling factors. Bottom row: transported longitudinal sequence in the target space. Red line indicates reference ventricles boundary.

\begin{tabular}{|l||c|c|c|c|}
\hline \multicolumn{1}{|c||}{} & \multicolumn{4}{c|}{ Jacobian determinant } \\
\hline Scaling factor & 0.5 & 1 & 2 & 3 \\
\hline \hline Source Space & 1.052 & 1.074 & 1.091 & 1.106 \\
Schild's ladder (BCH) & $1.062(.8 \mathrm{e}-2)$ & $1.094(1 \mathrm{e}-2)$ & $1.119(1.3 \mathrm{e}-2)$ & $1.13(1.8 \mathrm{e}-2)$ \\
Pole ladder (BCH) & $1.052(1 \mathrm{e}-2)$ & $1.063(1.2 \mathrm{e}-2)$ & $1.075(1.3 \mathrm{e}-2)$ & $1.086(1.3 \mathrm{e}-2)$ \\
Reorientation & $1.038(.6 \mathrm{e}-2)$ & $1.039(1 \mathrm{e}-2)$ & $1.033(1.5 \mathrm{e}-2)$ & $1.02(2.1 \mathrm{e}-2)$ \\
Conjugate Action & $1.026(.2 \mathrm{e}-2)$ & $1.038(.3 \mathrm{e}-2)$ & $1.049(.4 \mathrm{e}-2)$ & $1.063(.4 \mathrm{e}-2)$ \\
Scalar Interpolation & $1.054(.59 \mathrm{e}-2)$ & $1.077(1.12 \mathrm{e}-2)$ & $1.092(1.7 \mathrm{e}-2)$ & $1.100(2.4 \mathrm{e}-2)$ \\
\hline \multicolumn{4}{|||}{} & \multicolumn{4}{|c|}{$\log$ Jacobian determinant } \\
\hline Scaling factor & 0.5 & 1 & 2 & 3 \\
\hline \hline Source Space & .419 & .63 & .834 & 1.03 \\
Schild's ladder (BCH) & $.49(5 \mathrm{e}-2)$ & $.8(6.1 \mathrm{e}-2)$ & $1.14(5.4 \mathrm{e}-2)$ & $1.47(5.7 \mathrm{e}-2)$ \\
pole ladder (BCH) & $.37(9.4 \mathrm{e}-2)$ & $.56(14 \mathrm{e}-2)$ & $.75(18 \mathrm{e}-2)$ & $.93(23 \mathrm{e}-2)$ \\
Reorientation & $.5(8.9 \mathrm{e}-2)$ & $.76(13 \mathrm{e}-2)$ & $1.02(19 \mathrm{e}-2)$ & $1.26(21 \mathrm{e}-2)$ \\
Conjugate Action & $.21(0.5 \mathrm{e}-2)$ & $.32(1.2 \mathrm{e}-2)$ & $.43(2 \mathrm{e}-2)$ & $.38(29 \mathrm{e}-2)$ \\
Scalar Interpolation & $.44(9.5 \mathrm{e}-2)$ & $.66(17 \mathrm{e}-2)$ & $.86(26 \mathrm{e}-2)$ & $1.03(36 \mathrm{e}-2)$ \\
\hline
\end{tabular}

Table 2: Average measures (standard deviation) of ventricular changes associated to the initial longitudinal deformations in the source space (first row), and to the deformations transported with the different methods in the target spaces. Pole ladder performs similarly to the scalar interpolation while transporting in addition the full SVF. 


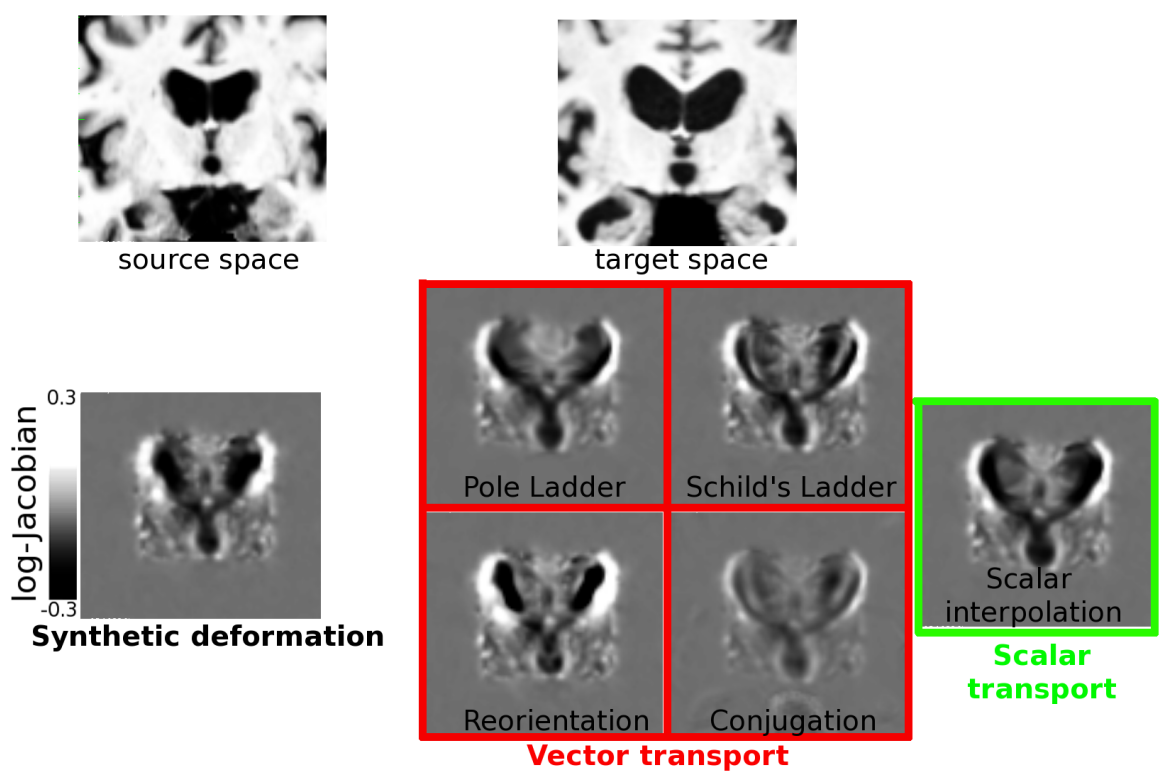

Fig. 7: Log-Jacobian maps of the transported deformation field from the source space $I_{0}$ to a patient's target space. Left: log-Jacobian map corresponding to the synthetic intra-subject deformation $\varphi_{1}$ in the source space. Right: log-Jacobian maps of the deformation in the target space transported thanks to: pole ladder, Schils's ladder, Reorientation, Conjugation, and scalar interpolation of the original log-Jacobian map. Schild's and pole ladders provide stable results which are consistent with the simple scalar transport.

from a broad range of academic institutions and private corporations, and subjects were recruited from over 50 sites across the U.S. and Canada. For up-to-date information, see www.adni-info.org.

\subsubsection{Longitudinal analysis}

Images corresponding to the baseline $I_{0}$ and the one-year follow-up $I_{1}$ scans were selected for 135 subjects affected by Alzheimer's disease from the ADNI database. For each subject $i$, the pairs of scans were rigidly aligned. The baseline was linearly registered to a reference template and the parameters of the transformation were applied to $I_{1}^{i}$. Finally, for each subject, the longitudinal changes were measured by non-rigid registration using the LCC-Demons algorithm [11].

The resulting deformation fields $\varphi_{i}=\exp \left(v_{i}\right)$ were transported with the pole ladder (BCH scheme) in the template reference along the subject-to-template deformation. The group-wise longitudinal progression was modeled as the mean of the transported SVFs $v_{i}$. The areas of significant longitudinal changes were investigated by one-sample t-test on the group of log-Jacobian scalar maps corresponding to the transported deformations, in order to detect the areas of measured expansion/contraction significantly different from zero. 
For sake of comparison, the one sample t-statistic was tested on the subject specific longitudinal log-Jacobian scalar maps warped into the template space along the subject-to-template deformation. This is the classical transport used in tensor's based morphometry studies [4].

\subsubsection{Results}

Figure 8 shows a detail from the mean SVF from the transported one-year longitudinal trajectories. The field flows outward from the ventricles to indicate a pronounced enlargement. Moreover, we notice an expansion in the temporal horns of the ventricles as well as a consistent contracting flow in the temporal areas. The same effect can be statistically quantified by evaluating the areas where the log-Jacobian maps are statistically different from zero. The areas of significant expansion are located around the ventricles and spread in the CSF areas, while a significant contraction is appreciable in the temporal lobes, hippocampi, parahippocampal gyrus and in the posterior cingulate. The statistical result is in agreement with the one provided by the simple scalar interpolation of the longitudinal subject specific log-Jacobian maps. In fact we do not experience any substantial loss of localisation power by transporting SVFs instead of scalar log-Jacobian maps. However by parallel transporting we preserve also the multidimensional information of the SVFs that, as experienced in [12, potentially leads to more powerful voxel-by-voxel comparisons than the ones obtained with univariate tests on scalars.

\section{Conclusions and Perspectives}

In this study we proposed a novel framework for the transport of longitudinal deformations in a reference space from time series of images. The mathematical formulation was combined with an effective computational scheme in order to provide a solution for the transport of vector fields.

From the applicative point of view, the availability of multivariate features in a common space could provide novel information for the understanding of biological processes. Moreover, although designed for transporting vector quantities, the method showed also good results in transporting scalar measures, by preserving smoothness of the corresponding spatial maps and providing accurate numerical evaluations. This is an interesting feature which could increase the power in TBMlike group-wise statistical analysis as well as opening the way to reliable multivariate group-wise analysis. The high spatial resolution of the statistical results on the experiment on the real data suggests a high precision of the procedure in transporting the different subject-specific trajectories. When associating the proposed transport with specific frameworks for the estimation of longitudinal trajectories, we were able to consistently model the longitudinal changes in clinical populations by including multiple time points, and to compare the progression of different clinical groups on a multidimensional basis $[12$. As shown in the experimental section, the stability of the proposed ladder critically depends on the choice of the scaling factor.

Even though the proposed scheme seems to provide reasonable and stable results, further perspective studies are required in order to investigate the numerical 

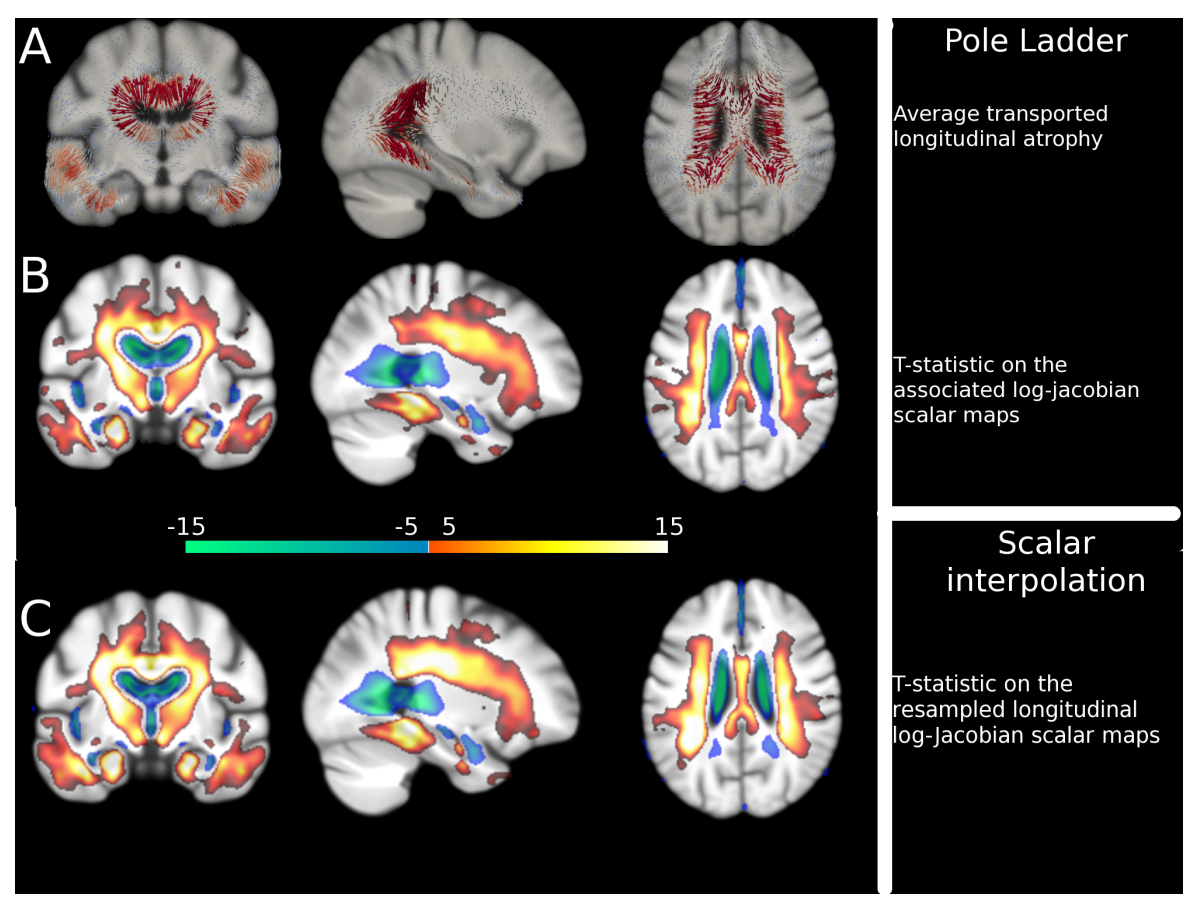

Fig. 8: One year structural changes for 135 Alzheimer's patients. A) Mean of the longitudinal SVFs transported in the template space with the pole ladder. We notice the lateral expansion of the ventricles and the contraction in the temporal areas. B) T-statistic for the correspondent log-Jacobian values significantly different from $0(p<0.001$ FDR corrected). C) T-statistic for longitudinal log-Jacobian scalar maps resampled from the subject to the template space. Blue color: significant expansion, Red color: significant contraction.

issues related to the step-size of the iterative computation. Finally, the comparison of the pole ladder with other computational schemes of the parallel transport, such as with the one proposed in 24, might shed more light on the theoretical and numerical properties of different methods of transport.

\section{Acknowledgment}

This was was partially supported by the ANR-blanc grant Karametria (2010-2013) (on a generic and extensible toolbox for feature-based morphometry in neuroimaging) from the French National research Agency and by the European Research Council through the ERC Advanced Grant MedYMA 2011-2018 (on Biophysical Modeling and Analysis of Dynamic Medical Images).

Data collection and sharing for this project was funded by the Alzheimer's Disease Neuroimaging Initiative (ADNI) (National Institutes of Health Grant U01 AG024904). ADNI is funded by the National Institute on Aging, the National 
Institute of Biomedical Imaging and Bioengineering, and through generous contributions from the following: Abbott; Alzheimer's Association; Alzheimer's Drug Discovery Foundation; Amorfix Life Sciences Ltd.; AstraZeneca; Bayer HealthCare; BioClinica, Inc.; Biogen Idec Inc.; Bristol-Myers Squibb Company; Eisai Inc.; Elan Pharmaceuticals Inc.; Eli Lilly and Company; F. Hoffmann-La Roche Ltd and its affiliated company Genentech, Inc.; GE Healthcare; Innogenetics, N.V.; IXICO Ltd.; Janssen Alzheimer Immunotherapy Research \& Development, LLC.; Johnson \& Johnson Pharmaceutical Research \& Development LLC.; Medpace, Inc.; Merck \& Co., Inc.; Meso Scale Diagnostics, LLC.; Novartis Pharmaceuticals Corporation; Pfizer Inc.; Servier; Synarc Inc.; and Takeda Pharmaceutical Company. The Canadian Institutes of Health Research is providing funds to support ADNI clinical sites in Canada. The grantee organization is the Northern California Institute for Research and Education, and the study is coordinated by the Alzheimer's Disease Cooperative Study at the University of California, San Diego. ADNI data are disseminated by the Laboratory for Neuro Imaging at the University of California, Los Angeles. This research was also supported by NIH grants P30 AG010129 and K01 AG030514.

\section{References}

1. Arnold, V.I.: Mathematical methods of classical mechanics. Springer, 60, (1989)

2. Arsigny, V., Commowick, O., Pennec, X., Ayache, N.: A Log-Euclidean framework for statistics on diffeomorphisms. In: Medical Image Computing and Computer-Assisted Intervention - MICCAI, vol. 9, pp. 924-931 (2006)

3. Ashburner, J., Friston, K.: Voxel-based morphometry - the methods. NeuroImage 11, 805-821 (2000)

4. Ashburner J, Ridgway GR.: Symmetric diffeomorphic modeling of longitudinal structural MRI. Front Neurosci. 2012;6:197. doi: 10.3389/fnins.2012.00197. Epub 2013 Feb 5.

5. Beg, M.F., Miller, M.I., Trouve, A., Younes, L.: Computing Large Deformation Metric Mappings via Geodesic Flows of Diffeomorphisms. Int. J. Comput. Vision 61(2), 139-157 (2005)

6. Bossa, M., Hernandez, M., Olmos, S.: Contributions to 3D diffeomorphic atlas estimation: Application to brain images. In: Medical Image Computing and Computer-Assisted Intervention - MICCAI, vol. 10, pp. 667-674 (2007)

7. Bossa, M., Zacur, E., Olmos, S.: On changing coordinate systems for longitudinal tensorbased morphometry. Spatio Temporal Image Analysis Workshop (STIA), 2010 (2010)

8. do Carmo, M.: Riemannian Geometry. Mathematics. Birkhäuser, Boston, Basel, Berlin (1992)

9. Duchateau, N.,De Craene, M., Pennec, X., Merino, B., Sitges, M., Bijnens, B.: Which Reorientation Framework for the Atlas-Based Comparison of Motion from Cardiac Image Sequences? Spatio-temporal Image Analysis for Longitudinal and Time-Series Image Data, Lecture Notes in Computer Science Volume 7570, 2012, pp 25-37

10. Kheyfets, A., Miller, W., Newton, G.: Schild's Ladder parallel transport for an arbitrary connection. International Journal of Theoretical Physics 39(12), 41-56 (2000)

11. Lorenzi, M., Ayache, N., Frisoni, G.B., Pennec, X.: LCC-Demons: a robust and accurate symmetric diffeomorphic registration algorithm. Neuroimage. 2013 May 16.

12. Lorenzi, M., Ayache, N., Frisoni, G.B., Pennec, X.: Mapping the effects of A $\beta_{1-42}$ levels on the longitudinal changes in healthy aging: hierarchical modeling based on stationary velocity fields. In: MICCAI, LNCS, pp. 663-670. Springer (2011)

13. Lorenzi, M., Ayache, N., Pennec, X.: Schild's Ladder for the parallel transport of deformations in time series of images. In: Information Processing in Medical Imaging - IPMI, vol. 22, pp. 463-474 (2011)

14. Lorenzi, M., Pennec, X.: Geodesics, parallel transport \& one-parameter subgroups for diffeomorphic image registration. International Journal of Computer Vision - IJCV (2012) 
15. Lorenzi, M., and Pennec X.: Parallel Transport with Pole Ladder: Application to Deformations of time Series of Images. International Conference on Geometric Science of Information - GSI (2013)

16. Misner, C.W., Thorne, K.S., Wheeler, J.: Gravitation. W.H. Freeman and Compagny (1973)

17. Postnikov, M.M.: Geometry VI: Riemannian Geometry. Encyclopedia of mathematical science. Springer (2001)

18. Qiu, A., Albert, M., Younes, L., Miller, M.: Time sequence diffeomorphic metric mapping and parallel transport track time-dependent shape changes. Neuroimage 45(1) (2009)

19. Qiu, A., Younes, L., Miller, M., Csernansky, J.: Parallel transport in diffeomorphisms distinguish the time-dependent pattern of hippocampal surface deformation due to healthy aging and dementia of the Alzheimer's type. Neuroimage 40(1) (2008)

20. Rao, A., Chandrashekara, R., Sanchez-Hortiz, G., Mohiaddin, R., aljabar, P., Hajnal, J., Puri, B., Rueckert, D.: Spatial trasformation of motion and deformation fields using nonrigid registration. IEEE Transactions on Medical Imaging 23(9) (2004)

21. Riddle, W.R., Li, R., Fitzpatrick, J.M., DonLevy, S.C., Dawant, B.M., Price, R.R.: Characterizing changes in MR images with color-coded Jacobians. Magn Reson Imaging 22(6), 769-777 (2004)

22. Schild, A.: Tearing geometry to pieces: More on conformal geometry. unpublished lecture at Jan. 191970 Princeton Univesity relativity seminar (1970)

23. Trouvé, A.: Diffeomorphisms groups and pattern matching in image analysis. Int. J. Comput. Vision 28(3), 213-221 (1998)

24. Vercauteren, T., Pennec, X., Perchant, A., Ayache, N.: Symmetric Log-domain diffeomorphic registration: A Demons-based approach. In: Proceedings of Medical Image Computing and Computer Assisted Intervention (MICCAI), vol. 5241, pp. 754-761 (2008)

25. Younes, L.: Jacobi fields in groups of diffeomorphisms and applications. Q. Appl. Math pp. 113-134 (2007)

26. Yushkevich, P., Piven, J., Hazlett, H., Smith, R., Ho, S., Gee, J., Gerig, G.: User-guided $3 \mathrm{D}$ active contour segmentation of anatomical structures: Significantly improved efficiency and reliability. Neuroimage 31(3) (2006) 